\title{
SOP Somatische Diagnostik bei Verdacht auf immunologische Psychosen
}

Ludger Tebartz van Elst, Dominique Endres

\begin{abstract}
Immunologische Psychosen stellen eine Gruppe von Krankheitsbildern dar, welche zunehmend als differenzialdiagnostische Erwägung insbesondere bei psychotischen Störungsbildern diskutiert werden. Die Prognose dieser Unterform psychiatrischer Störungsbilder hängt von einer frühzeitigen Diagnose ab. Für eine umfassende Beurteilung sind der klinische Phänotyp und eine Reihe von Zusatzuntersuchungen erforderlich.
\end{abstract}

\section{Einleitung}

Die Annahme, dass psychotische Störungsbilder durch entzündliche Prozesse des Gehirns verursacht sein könnten wurde bereits in der ersten Hälfte des letzten Jahrhunderts formuliert [1]. Angestoßen durch neue Erkenntnisse der letzten Dekaden aus dem Themenbereich der limbischen Encephalitiden in der Neurologie [2,3] wird eine mögliche Rolle autoimmunologischer Prozesse auch in der Genese psychiatrischer Störungen - insbesondere von Psychosen - zunehmend diskutiert $[4,5]$. In diesem Zusammenhang wird vorgeschlagen, bei der ätiopathogenetischen Einordnung der wesentlich syndromal konzeptualisierten, psychiatrischen Störungsbilder zunehmend nach kausalen Kriterien zwischen primär-idiopathischen Varianten (z.B. im Sinne einer genuinen Schizophrenie) und sekundär-symptomatischen Varianten (z. B. im Sinne einer immunologischen schizophreniformen Psychose) zu unterscheiden [6]. Im Zentrum der Aufmerksamkeit stehen dabei bislang überwiegend Autoantikörper-vermittelte autoimmunologische Prozesse. Die wichtigsten der aktuell bekannten gegen Hirngewebe gerichteten Autoantikörper im Kontext der immunologischen Psychosen sind in > Tab. 1 zusammengefasst.
Wesentlicher klinischer Anknüpfungspunkt für den Verdacht einer sekundären, immunologischen Genese einer Psychose ist dabei der akute bis subakute Beginn einer oft im Detail atypisch anmutenden, polymorphen, psychotischen Symptomatik, die häufig begleitet wird von unverhältnismäßigen neurokognitiven Symptomen, Bewegungsstörungen (katatone Symptome, Dyskinesien) oder gar klaren neurologischen Symptomen, etwa im Sinne von Bewusstseinsstörungen, epileptischen Anfällen, Aphasien, Dysarthrien, autonomen Funktionsstörungen etc. Ist die Symptomatik zudem im Kontext einer fieberhaften Erkrankung aufgetreten, sollte die Differenzialdiagnose unbedingt erwogen werden. Insgesamt können aus klinischer Perspektive härtere Warnzeichen von weicheren („red flags“) unterschieden werden wie in > Tab. 2 zusammengefasst.

\section{PRAXISTIPP}

Bei einer akut-subakuten, polymorphen, psychotischen Symptomatik sollte differenzialdiagnostisch an eine immunologische Psychose gedacht werden - vor allem dann, wenn folgende Warnzeichen gegeben sind:

- katatone Symptome

- Dyskinesien (orofazial, an den Extremitäten)

- epileptische Anfälle, faziobrachiale dystone Anfälle

- Vigilanzstörungen

- autonome Instabilität

- Sprachstörungen: Aphasie und Dysarthrie

- fokale neurologische Defizite

- Kopfschmerzen/Fieber (insbesondere prodromales Auftreten) 


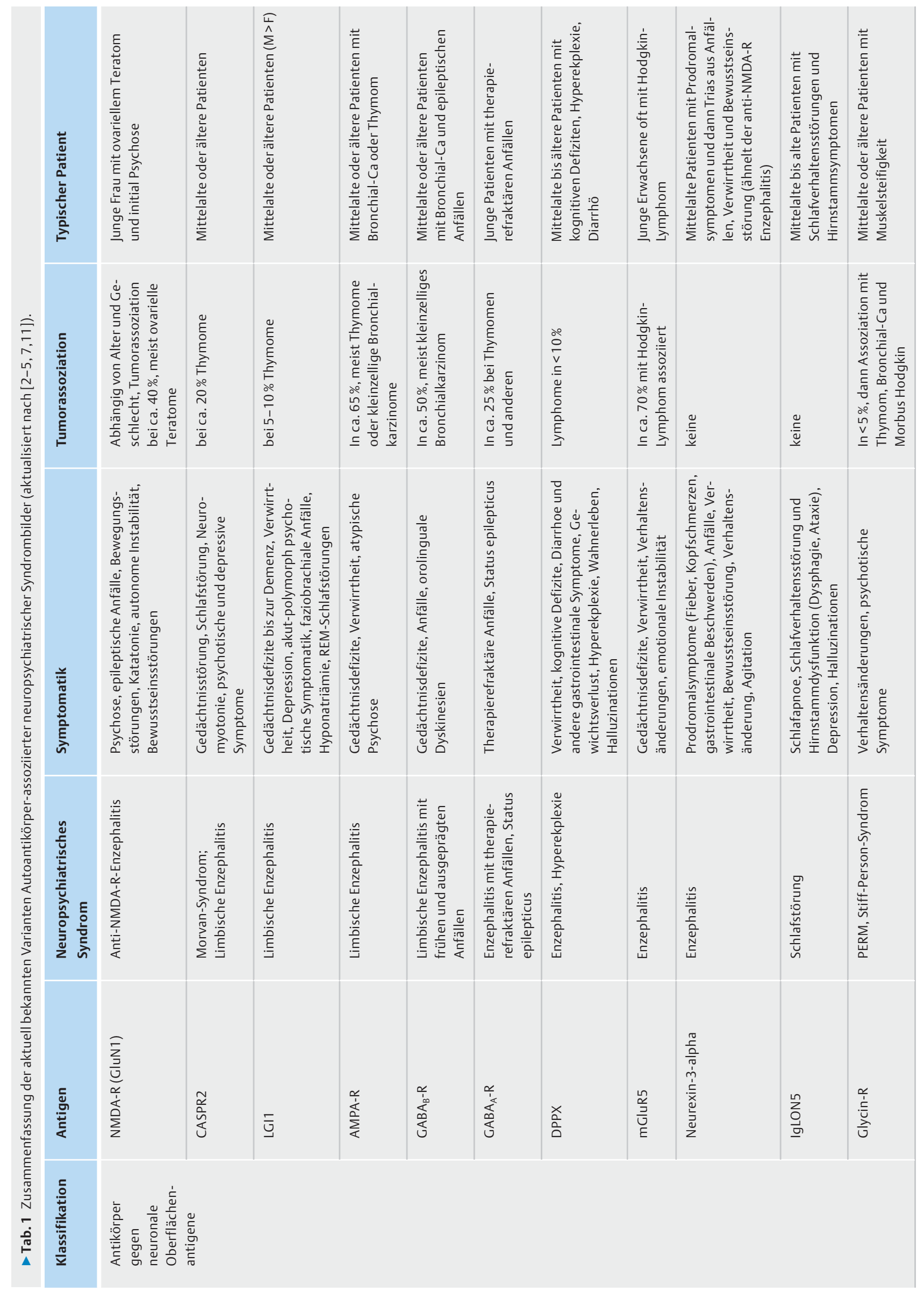




\begin{tabular}{|c|c|c|c|c|c|}
\hline 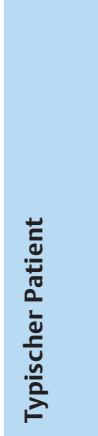 & 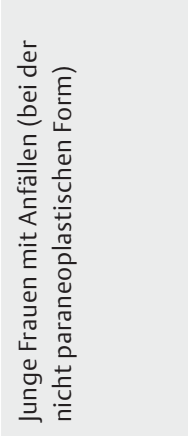 & 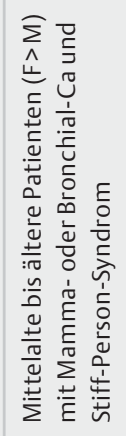 & 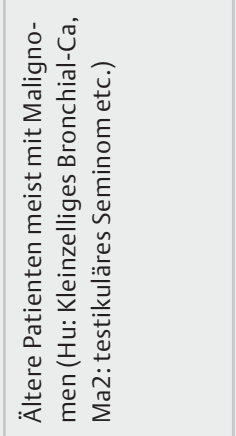 & 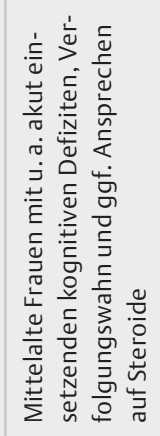 & 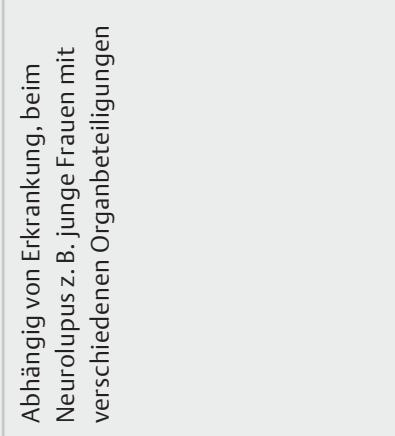 \\
\hline 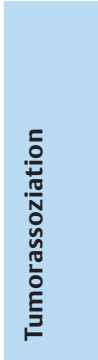 & 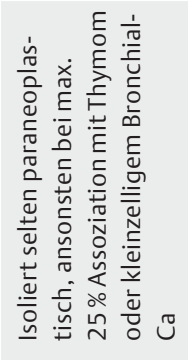 & 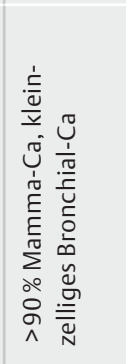 & 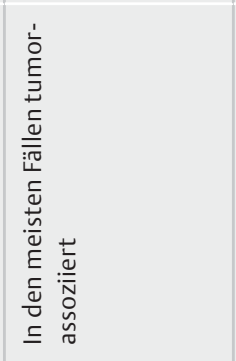 & 喜 & 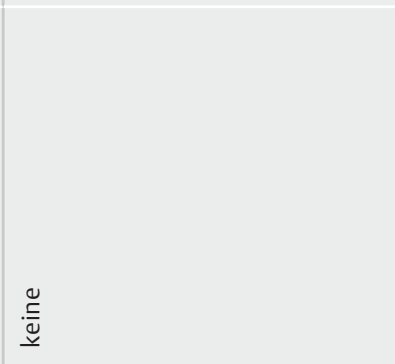 \\
\hline & 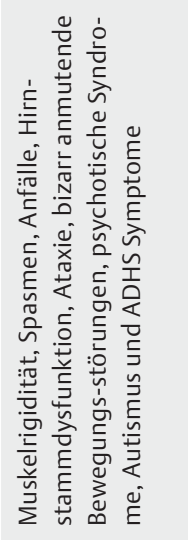 & 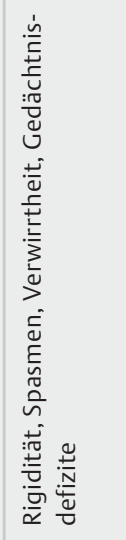 & 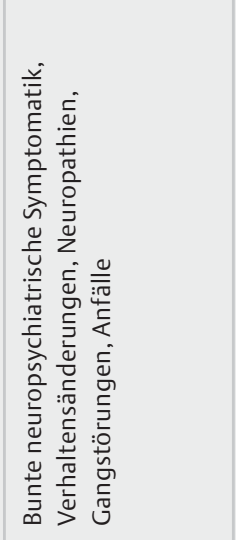 & 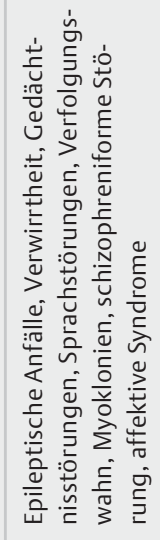 & 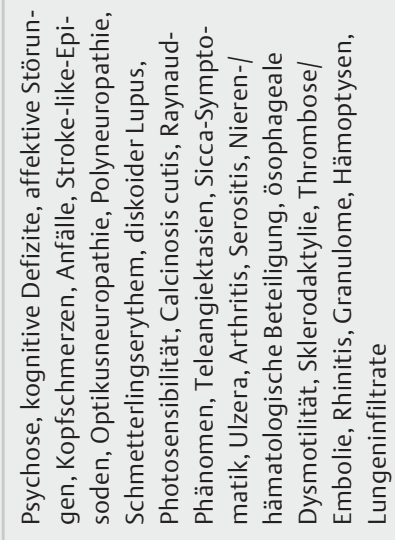 \\
\hline 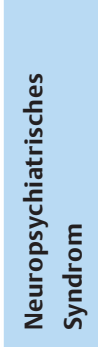 & 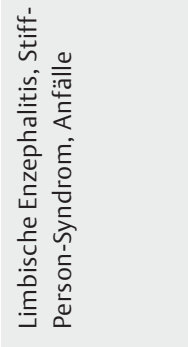 & 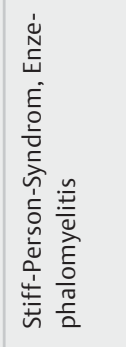 & 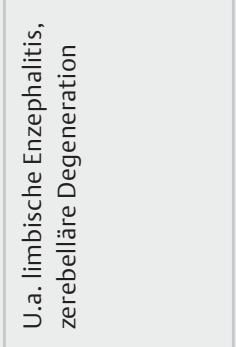 & 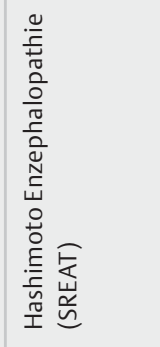 & 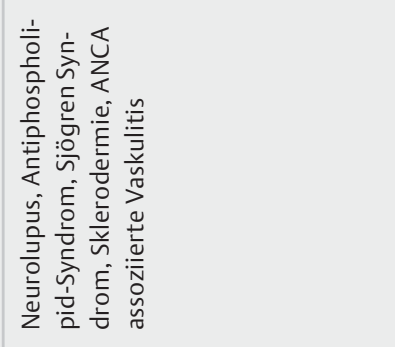 \\
\hline 㤐 & 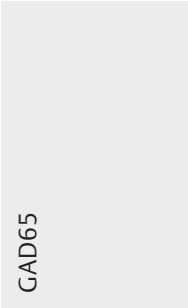 & 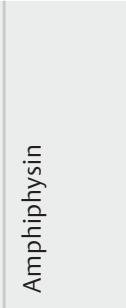 & 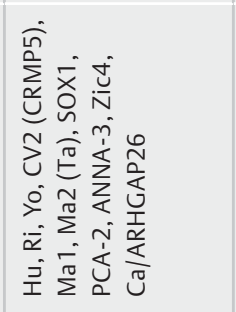 & 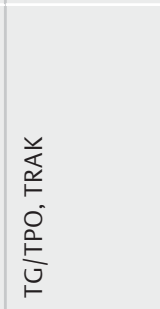 & 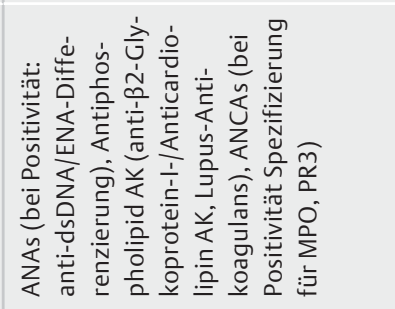 \\
\hline 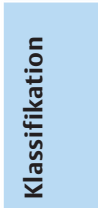 & 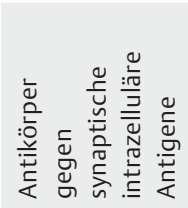 & & 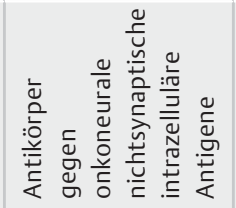 & 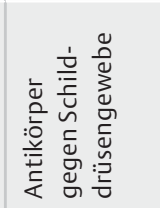 & 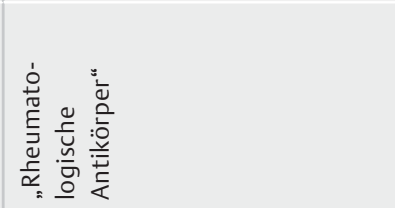 \\
\hline
\end{tabular}


- Tab. 2 „Harte“ und „weiche“ Warnzeichen, die an eine autoimmune Enzephalitis bzw. immunologische Psychose denken lassen sollten (zitiert nach [8]: https://www.awmf.org/uploads/tx_szleitlinien/038-009l_S3_Schizophrenie_2019-03.pdf).

\begin{tabular}{|c|c|}
\hline „Harte“ Warnzeichen & „Weiche“ Warnzeichen \\
\hline $\begin{array}{l}\text { Lymphozytäre Liquor-Pleozytose ohne Hinweise auf eine } \\
\text { infektiöse Ursache }\end{array}$ & Quantitative Bewusstseinsstörungen \\
\hline Epileptische Anfälle & Bewegungsstörung oder Stand- und Gangunsicherheit \\
\hline Faziobrachiale dystone Anfälle & Autonome Instabilität \\
\hline MRT-Auffälligkeiten (z. B. mesiotemporale Hyperintensitäten) & Fokalneurologische Defizite, inkl. Aphasie oder Dysarthrie \\
\hline \multirow{5}{*}{$\begin{array}{l}\text { EEG-Auffälligkeiten (Grundrhythmus-Verlangsamung, } \\
\text { epilepsietypische Muster, holozephaler extremer Delta-Brush } \\
\text { (Beta-Delta-Komplexe, welche aus bilateraler Delta-Aktivität } \\
\text { mit } 1-3 \mathrm{~Hz} \text { und aufgelagerter Beta-Aktivität mit } 20-30 \mathrm{~Hz} \\
\text { bestehen), die nicht anderweitig erklärt werden können. Der } \\
\text { extreme Delta-Brush bei nicht-neugeborenen Personen scheint } \\
\text { häufig bei NMDAR-Autoimmunenzephalitis aufzutreten, wobei } \\
\text { die Spezifität nicht klar ist }\end{array}$} & $\begin{array}{l}\text { Schnelles Fortschreiten der psychotischen Symptome trotz } \\
\text { psychopharmakologischer Therapie }\end{array}$ \\
\hline & Hyponatriämie \\
\hline & Ätiologisch ungeklärte Kopfschmerzen \\
\hline & Katatonie \\
\hline & Andere komorbide Autoimmunerkrankungen \\
\hline
\end{tabular}

\section{Internationale Konsensbemühungen bei der Operationalisierung immunologischer Psychosen}

Das oben beschriebene klinische Bild repräsentiert einen klassischen „neuropsychiatrischen Phänotyp“, der unmittelbar den Verdacht einer organischen Genese der Symptomatik begründen wird, wenn er in seiner ganzen Fülle auftritt, etwa in Kombination mit epileptischen Anfällen. Die klinische Erfahrung zeigt aber, dass in vielen Fällen auch nur wenige oder einzelne der Warnzeichen auftreten. Auch wenn epileptische Anfälle oder Dyskinesien als Nebenwirkungen einer zuvor initiierten psychotropen Medikation fehlgedeutet werden, kann auch eine klar neuropsychiatrische Präsentation im Sinne einer primär-idiopathischen Verursachung fehlgedeutet werden [6].

Vor dem Hintergrund dieser Schwierigkeit bei der klinischen Identifizierung entsprechender Fälle wurde im Rahmen internationaler Konsensbemühungen das klinische Bild einer möglichen, wahrscheinlichen und sicheren immunologischen bzw. autoimmunen Psychose operationalisiert (vgl. die folgenden Infokästen; modifiziert und übersetzt von den Autoren nach [7]).

\section{MÖGLICHE IMMUNOLOGISCHE PSYCHOSE Operationalisierung einer möglichen immunologischen Psychose nach [7]}

Der Patient muss gegenwärtig an psychotischen Symptomen mit abruptem Beginn (schnelle Progression < 3 Monate) leiden und wenigstens einen der folgenden Punkte aufweisen:

- gegenwärtig oder kürzlich diagnostizierte Tumorerkrankung

- Bewegungsstörung (Katatonie oder Dyskinesie)

- unerwünschte Reaktion auf Antipsychotika, die den Verdacht auf ein malignes neuroleptisches Syndrom (Rigidität, Hyperthermie oder erhöhte Kreatinkinase) aufkommen lassen

- schwere oder unverhältnismäßige kognitive Dysfunktion

- ein vermindertes Bewusstseinsniveau

- das Auftreten von Krampfanfällen, die nicht durch eine zuvor bekannte Krampfanfallerkrankung erklärt werden können

- eine klinisch bedeutsame autonome Funktionsstörung (abnormaler oder unerwartet schwankender Blutdruck, Temperatur oder Herzfrequenz) 
Tab.3 Spezifische Empfehlungen zur organischen Diagnostik bei immunologischen Psychosen (gemäß [7-9]).

\begin{tabular}{|c|c|c|c|c|}
\hline Modalität & $\begin{array}{l}\text { Konsens- } \\
\text { Empfehlung } \\
\text { (nach [7]) }\end{array}$ & S3-Leitlinien [8] & $\begin{array}{l}\text { Freiburger Protokoll } \\
\text { (partiell beschrieben in [9]) }\end{array}$ & Anmerkungen \\
\hline EEG & $\begin{array}{l}\text { Keine } \\
\text { Spezifikation }\end{array}$ & Keine Spezifikation & 32-Kanal klinisches Routine EEG & \\
\hline MRT & $\begin{array}{l}\text { FLAIR- } \\
\text { Sequenzen }\end{array}$ & $\begin{array}{l}\text { T1-, T2-, FLAIR-Sequenzen, bei Auffäl- } \\
\text { ligkeiten weiterführende Diagnostik } \\
\text { mit Kontrastmittel-MRT (bei nicht Ver- } \\
\text { fügbarkeit oder Kontraindikationen für } \\
\text { eine MRT Untersuchung: CCT) }\end{array}$ & $\begin{array}{l}\text { FLAIR axial + FLAIR coronar “hippocampal } \\
\text { view”, T2 coronar, DWI axial und coronar, T2* } \\
\text { axial oder SWI, T1-MPRAGE (1x1x1 mm); bei } \\
\text { spezifischen Verdacht mit Kontrastmittel }\end{array}$ & $\begin{array}{l}\text { Eine CT Untersu- } \\
\text { chung erscheint } \\
\text { nicht geeignet, um } \\
\text { immunologische } \\
\text { Prozesse mit hin- } \\
\text { reichender Sicher- } \\
\text { heit zu beurteilen }\end{array}$ \\
\hline $\begin{array}{l}\text { Liquor } \\
\text { (CSF) }\end{array}$ & $\begin{array}{l}\text { Routine und } \\
\text { antineuronale } \\
\text { Antikörper }\end{array}$ & Keine Spezifikation & $\begin{array}{l}\text { Routine und antineuronale Antikörper (bei } \\
\text { spezifischem Verdacht kann eine Bestimmung } \\
\text { der Antikörperbindung auf nativen murinen } \\
\text { zerebralen Gewebsschnitten hilfreich sein) }\end{array}$ & \\
\hline Labor & $\begin{array}{l}\text { Anti-neuronale } \\
\text { Antikörper }\end{array}$ & $\begin{array}{l}\text { Obligat: } \\
\text { Differenzialblutbild } \\
\text { Nüchternblutzucker und ggf. } \mathrm{HbA}_{1 \mathrm{c}} \\
\text { GPT, Gamma-GT } \\
\text { Kreatinin/eGFR } \\
\text { Natrium, Kalium, Calcium } \\
\text { BSG/CRP } \\
\text { Schilddrüsenparameter (initial TSH) } \\
\text { Fakultativ: } \\
\text { Creatininkinase (CK), } \\
\text { Rheumatologisches Labor, } \\
\text { Eisen- und Kupferstoffwechsel, } \\
\text { Vitamin }{ }_{1}, B_{6}, B_{12} \text {, } \\
\text { Serologie für wichtige Infektionser- } \\
\text { krankungen (HIV, Hepatitis, Lues, etc.) } \\
\text {... }\end{array}$ & $\begin{array}{l}\text { Routine: Blutbild, Elektrolyte, Nieren/Leber- } \\
\text { werte, Schilddrüsenhormone, CRP, HBA } 1 \text {, Li- } \\
\text { pide, U-Status, Vitamin } \text { B }_{12} \text {, Folsäure, Vitamin } \\
\text { D, Selen; Lues-, Borrellienserologie, anti-TPO, } \\
\text { TG and TRAK, Rheuma-Screening: IgA, IgG, } \\
\text { IgM, Immunfixation; CH 50, C3, C4, C3 D, } \\
\text { Antiphospholipid AK, ANA-IF-Screening (falls } \\
\text { ANA Screening positiv: ENA Spezifikation: an- } \\
\text { ti-dsDNA, Histone, rib. P-Proteine, snRNP, Sm, } \\
\text { SS-A/Ro, Ro-52, SS-B/La, Scl-70, PM-SCL, Jo-1, } \\
\text { Zentromere, PCNA; Nukleosomen, DFS-70), } \\
\text { ANCA-IF-Screening (falls positiv Testung auf } \\
\text { MPO, PR3), Alpha-Galaktosidase, } \\
\text { Antineuronale Antikörper gegen: Hu, Yo, Ri, } \\
\text { CV2 /CRMP5, Amphiphysin, Ma1, Ma2, SOX1, } \\
\text { GAD65, Tr(DNER), Zic4; VGKC (LGI1, CASPR2), } \\
\text { GABA B, NMDA-R, AMPA } 1 \text { und AMPA 2; Aqua- } \\
\text { Porin4, MOG }\end{array}$ & $\begin{array}{l}\text { Hyponatriämie } \\
\text { kann auf LGI1 Enze- } \\
\text { phalitis hinweisen }\end{array}$ \\
\hline FDG-PET & $\begin{array}{l}\text { Keine } \\
\text { Spezifikation }\end{array}$ & Keine Spezifikation? & Bei spezifischem Verdacht & \\
\hline
\end{tabular}

Kann nach dieser Operationalisierung die Diagnose einer möglichen immunologischen Psychose gestellt werden, so werden gemäß Konsensempfehlungen folgende diagnostische Untersuchungen im Sinne eines Standardprozederes empfohlen: EEG, MRT, Liquoranalyse (inklusive Testung auf antineuronale Antikörper) und verschiedene Laboruntersuchungen (vgl. > Tab. 3).

Bei der Betrachtung der verschiedenen Empfehlungen muss berücksichtigt werden, dass die Trennung der psychiatrischen Disziplinen von der somatischen Medizin in den verschiedenen Ländern der Welt unterschiedlich weit fortgeschritten ist. So kann es in vielen Ländern durchaus schwierig sein, im psychiatrischen Versorgungssetting überhaupt eine EEG- oder Liquoruntersuchung zu organisieren, während dies in den meisten deutschen psychiatrischen Versorgungskrankenhäusern möglich ist. Diese internationale Perspek- tive erklärt die gestufte Vorgehensweise der internationalen Konsensgruppe.

Merke
Bei einer möglichen immunologischen Psychose
sollten nach internationalem Konsens MRT-, EEG-
und Liquoruntersuchungen durchgeführt werden.
Auf der Grundlage der Untersuchungsergebnisse sollte
dann die Diagnose einer wahrscheinlichen oder defini-
tiven Psychose gestellt oder zurückgewiesen werden.




\section{IMMUNOLOGISCHE PSYCHOSE}

Operationalisierung einer wahrscheinlichen immunologischen Psychose (nach [7])

Der Patient muss eine mögliche Autoimmunpsychose (s.o.) haben und

- mindestens einen der folgenden Punkte:

- Liquor-Pleozytose von > 5 weißen Blutkörperchen per $\mu \mathrm{l}$

- fokale bilaterale Hirnanomalien (in den T2-gewichteten FLAIR MRT-Sequenzen) im Bereich der medialen Temporallappen

- oder 2 der folgenden Punkte:

- EEG: Enzephalopathische Veränderungen (d.h. Spitzen, Spike-Wave-Aktivität oder rhythmische Verlangsamung [intermittierende rhythmische Delta- oder ThetaAktivität]), fokale Veränderungen oder „Extreme Delta-Brush“

- oligoklonale Banden im Liquor oder erhöhter IgG-Index

- Vorhandensein eines anti-neuronalen Antikörpers im Serum (der mit einem zellbasierten Assay nachgewiesen wurde)

Nach Ausschluss von Alternativdiagnosen.

\section{Operationalisierung einer sicheren} immunologischen Psychose (nach [7])

Der Patient erfüllt die Kriterien einer wahrscheinlichen immunologischen Psychose und es wurden antineuronale IgG-Antikörper im CSF nachgewiesen.

\section{Pragmatische Aspekte in der Diagnostik möglicher immunologischer Psychosen}

In der klinischen Praxis müssen die diagnostischen Maßnahmen meist in Kooperation mit der Neurologie durchgeführt werden. Vor diesem Hintergrund erscheint es auch sinnvoll, mit der Operationalisierung einer möglichen Autoimmunenzephalitis nach aktuellem internationalem neurologischen Konsens vertraut zu sein (s. Kasten). Aus der Darstellung wird ersichtlich, dass aus neurologischer Perspektive das Verlaufskriterium einer akuten/subakuten Dynamik entscheidend ist, um bei letztlich jedweder relevanten neurokognitiven, psychischen oder persönlichkeitsalterierenden Symptomatik eine umfassende Diagnostik inklusive MRT und Liquoruntersuchung zu indizieren. Lediglich auf die EEG-Untersuchung wird nicht explizit, sondern nur implizit im Kontext der Erwähnung der Epilepsiediagnostik abgehoben. Insbesondere im Hinblick auf die kritische Durchführung einer Untersuchung des zerebralen Liquors besteht jedoch Einvernehmen.

\section{MÖGLICHE AUTOIMMUNENZEPHALITIS}

Definition einer möglichen Autoimmunenzephalitis nach aktuellen neurologischen Konsenskriterien [8, 10]

Die Diagnose erscheint möglich, wenn alle 3 der folgenden Kriterien zutreffend sind:

1. Subakuter Beginn (schnelle Progression innerhalb <3 Monaten) von:

- Merkfähigkeitsstörung

- qualitativer oder quantitativer Bewusstseinsstörungen

- Lethargie

- Wesensänderung/Persönlichkeitsveränderungen oder

- anderer psychischer Symptome

2. Mindestens einer der folgenden Punkte:

- neu aufgetretene fokale neurologische Defizite

- neu aufgetretene epileptische Anfälle

- lymphozytäre Pleozytose im Liquor $(>5$ Zellen/ $\mu l)$
- MRT-Merkmale, die auf eine Enzephalitis hindeuten: hyperintenses MRT-Signal in T2-oder FLAIR-Sequenzen, mesiotemporal betont (limbische Enzephalitis) oder in multifokalen Bereichen, welche die graue Substanz, die weiße Substanz oder beides umfassen.

3. Ausschluss anderer Krankheitsursachen wie infektiöse Enzephalitis (neurotrope Viren: z. B. CMV, EBV, HSV, Influenza, Masern, Mumps, Röteln, VZV; andere Krankheitserreger: z. B. Borrelien, Chlamydien, Mykoplasmen, Candida albicans und Toxoplasma gondii) oder Sepsis, rheumatische Erkrankungen (z. B. Lupus erythematodes, Sarkoidose), metabolische und toxische Enzephalopathien (z. B. hepatisch, renal), mitochondriale Erkrankungen, zerebrovaskuläre Erkrankungen, Tumoren, Creutzfeldt-Jakob-Erkrankung. 


\section{Bewertung der zur Verfügung stehenden diagnostischen Verfahren}

Aus klinischer Sicht sind insbesondere die EEG- und Liquoruntersuchungen von hoher Bedeutung für die Einschätzung der Wahrscheinlichkeit, dass im konkreten Fall aus ursächlicher Sicht eine immunologische Psychose vorliegen könnte. Zwar können in Einzelfällen sowohl MRT als auch EEG und Liquoruntersuchungen bei Patienten mit immunologischen Psychosen unauffällig sein, nach den aktuell vorliegenden Daten aus Fallsammlungen ist dies aber beim MRT deutlich häufiger der Fall als beim Liquor und EEG-Befund.

Finden sich in der derart gestalteten Diagnostik keine eindeutigen Befunde, die die Diagnose einer klinisch sicheren immunologischen Psychose begründen, so kann eine FDG-PET-Untersuchung ein weiteres hilfreiches Instrument zur Objektivierung eines Befundes im Sinne einer immunologischen Enzephalitis sein [11, 16]. Auch können bei negativem Befund bei der Antikörpersuche im Serum und Liquor Spezialuntersuchungen auf fixierten/unfixierten Mäusehirnschnitten helfen, bislang noch unbekannte Antikörper zu detektieren, die sich dann meist gegen neuronale oder auch vaskuläre zerebrale Strukturen richten [12]. Auch kann insbesondere dann, wenn Antikörper mit spezifischer Assoziation zu Tumorleiden gefunden werden, eine Tumorsuche indiziert sein. $\mathbf{A b} \mathbf{\text { b }} \mathbf{1}$ illustriert das pragmatische Vorgehen bei der Diagnostik im klinischen Alltag.

\begin{tabular}{|c|}
\hline Labordiagnostik \\
\hline $\begin{array}{l}\text { Blutbild, Na, K, Kreatinin, GOT, GPT, gamma-GT, } \\
\text { CRP/BSG, TSH, fT3, fT4, TPO-Ak, TG-Ak }\end{array}$ \\
\hline$\downarrow$ \\
\hline apparative Zusatzdiagnostik \\
\hline $\begin{array}{l}\text { cMRT, EEG, neuropsychologische Testung } \\
\text { (mind. Bedsidetestung) }\end{array}$ \\
\hline$\downarrow$ \\
\hline Liquorpunktion \\
\hline $\begin{array}{l}\text { Basisdiagnostik, antineuronale Antikörper gegen } \\
\text { neuronale Zelloberflächenantigene, intrazelluläre } \\
\text { synaptische Antigene bzw. intrazelluläre onkoneuro- } \\
\text { nale Antigene }\end{array}$ \\
\hline$\downarrow$ \\
\hline ggf. FDG-PET \\
\hline ggf. Tumorsuche \\
\hline
\end{tabular}

Abb. 1 Pragmatisches Vorgehen bei klinischem Verdacht auf autoimmune Psychose (nach [11]).

\section{Therapeutische Implikationen}

Bei Diagnose einer immunologischen Psychose sollte nach allgemeinem klinischem Konsens eine Immuntherapie erwogen werden.

\section{Merke}

Die Behandlungsversuche der Immuntherapie bei immunologischen Psychosen sind off-label und benötigen entsprechend eine sorgfältige Aufklärung.

Initial wird oft eine Steroidpulsbehandlung eingesetzt (mit z.B. 500-1000 mg Methylprednisolon über 3-5 Tage). Zu den Erstlinientherapien zählen auch die Plasmapherese und intravenöse Immunglobuline.

Zu den Zweitlinienverfahren gehören Rituximab und Cyclophosphamid, wobei Rituximab zunehmend wichtiger wird [9,11-15]. Während ein Therapieversuch mit Kortison dabei auch im klassischen Setting der Psychiatrie meist gut umgesetzt werden kann, muss bei weiterer Therapieeskalation die Zusammenarbeit mit der Neurologie, Immunologie und Nephrologie gesucht werden. Ohnehin empfiehlt es sich, interdisziplinäre Strukturen zur umfassenden gemeinsamen diagnostischen und therapeutischen Behandlungsplanung zu etablieren, da es sich bei vielen der betroffenen Fälle um oft recht komplexe und schwer zu behandelnde Patienten handelt.

\section{Cave}

Ein fehlendes Ansprechen auf einen Behandlungsversuch mit Kortison schließt die Diagnose einer immunologischen Psychose nicht sicher aus.

Gleichzeitig muss ebenfalls betont werden, dass die Diagnose einer immunologischen Psychose nicht bedeutet, dass eine klassische symptomatische medikamentöse Therapie mit den verschiedenen psychotropen Medikamenten (Neuroleptika, Antikonvulsiva, Benzodiazepine, Antidepressiva etc.) nicht erfolgreich sein kann. Auch wenn extrapyramidal-motorische Symptome als Nebenwirkung einer Therapie mit Neuroleptika als Warnsignal für das Vorliegen einer immunologischen Psychose gelten, so kann daraus sicher nicht geschlossen werden, dass Neuroleptika bei anderen Patienten mit entsprechenden sekundären Psychosen nicht sehr gut wirken können und auch gut vertragen werden. Hier wird die klinische Forschung der kommenden Dekaden sicher noch viele interessante Ergebnisse hervorbringen.

\section{Merke}

Für viele Menschen mit immunologischer Psychose hat eine Immuntherapie zu sehr weitreichenden klinischen Besserungen bis hin zu fast kompletten Heilungen des psychotischen Prozesses geführt $[6,9,17]$. 


\section{Interessenkonflikt}

Die Autorinnen/Autoren geben an, dass kein Interessenkonflikt besteht.

\section{Autorinnen/Autoren}

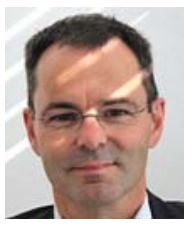

\section{Ludger Tebartz van Elst}

Prof. Dr. Stellvertretender Ärztlicher Direktor und Leitender Oberarzt, Leiter der Sektion experimentelle Neuropsychiatrie an der Klinik für Psychiatrie und Psychotherapie an der Uniklinik Freiburg. Forschungsschwerpunkte: Neurobiologie und Psychotherapie autistischer und schizophreniformer Syndrome, Affektive Neurowissenschaft, Visuelle Neurowissenschaft, Brain Imaging, MRI Morphometrie, EEG/fMRI, MR-Spektroskopie, Neurophilosophie: Neurobiologie der Freiheit.

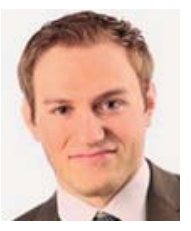

\section{Dominique Endres}

PD. Dr. Oberarzt in der Klinik für Psychiatrie und Psychotherapie an der Uniklinik Freiburg. Klinisch-wissenschaftlicher Schwerpunkt: Immunologische Enzephalopathien in der Psychiatrie, Zwangsstörungen, Entwicklungsstörungen, Bildgebung, Liquormarker.

\section{Korrespondenzadresse}

Prof. Dr. med. Ludger Tebartz van Elst

Klinik für Psychiatrie und Psychotherapie Sektion für experimentelle Neuropsychiatrie Universitätsklinikum Freiburg Hauptstr. 5

79104 Freiburg

tebartzvanelst@uniklinik-freiburg.de

\section{Acknowledgement}

D. Endres wird unterstützt durch das Berta-OttensteinProgramm für Advanced Clinician Scientists der medizinischen Fakultät der Universität Freiburg.

\section{Literatur}

[1] Roeder F. Über die serologische Diagnostik der Schizophrenie aus dem Liquor nach der Methode von Lehmann-Facius. Z Gesamte Neurol Psychiatr 1939; 165: 462-467

[2] Graus F, Titulaer M], Balu R et al. A clinical approach to diagnosis of autoimmune encephalitis. Lancet Neurol 2016; 15: 391-404. doi: 10.1016/S1474-4422(15)00401-9

[3] Dalmau J, Geis C, Graus F. Autoantibodies to Synaptic Receptors and Neuronal Cell Surface Proteins in Autoimmune Diseases of the Central Nervous System. Physiol Rev 2017; 97: 839-887. doi: 10.1152/physrev.00010.2016

[4] Endres D, Bechter K, Prüss $\mathrm{H}$ et al. Autoantibody-associated schizophreniform psychoses: clinical symptomatology. Nervenarzt 2019; 90: 547-563
[5] Tebartz van Elst L, Bechter K, Prüss H et al. Autoantibodyassociated schizophreniform psychoses: pathophysiology, diagnostics, and treatment. Nervenarzt 2019; 90: 745-761

[6] Tebartz van Elst L. Vom Anfang und Ende der Schizophrenie. Eine neuropsychiatrische Perspektive auf das Schizophreniekonzept Stuttgart: Kohlhammer Verlag; 2017

[7] Pollak TA, Lennox BR, Müller S et al. Autoimmune psychosis: an international consensus on an approach to the diagnosis and management of psychosis of suspected autoimmune origin. Lancet Psychiatry 2020; 7: 93-108

[8] AWMF. S3-Leitlinie Schizohrenie. https://www.awmf.org/ leitlinien/detail/II/038-009.html

[9] Endres D, Leypoldt F, Bechter K et al. Autoimmune encephalitis as a differential diagnosis of schizophreniform psychosis: clinical symptomatology, pathophysiology, diagnostic approach, and therapeutic considerations. Eur Arch Psychiatry Clin Neurosci 2020: doi: 10.1007/s00406-02001113-2

[10] Graus F, Titulaer M], Balu R et al. A clinical approach to diagnosis of autoimmune encephalitis. The Lancet Neurology 2016: 15: 391-404

[11] Tebartz van Elst L, Stich O, Endres D. Depressionen und Psychosen bei immunologischen Enzephalopathien. PSYCH up2date 2015; 9: 265-279

[12] Endres D, Prüss $\mathrm{H}$, Rauer $\mathrm{S}$ et al. Probable autoimmune catatonia with antibodies against cilia on hippocampal granule cells and highly suspicious cerebral FDG-positron emission tomography findings. Biol Psychiatry 2020; 87: e29-e31. doi: 10.1016/j.biopsych.2019.12.020

[13] Bien C. Immunvermittelte Erkrankungen der grauen ZNSSubstanz sowie Neurosarkoidose: S1-Leitlinie. Leitlinien für Diagnostik und Therapie in der Neurologie 2012. Available from: URL: http://www.dgn.org/leitlinien-online-2012/ inhalte-nach-kapitel/2396-II-32-2012-immunvermittelteerkrankungen-der-grauen-zns-substanz-sowie-neurosarkoidose.html

[14] Friese MA, Magnus T. Antikörper-assoziierte autoimmune Enzephalopathien. In: Brandt T, Diener HC, Gerloff C. Therapie und Verlauf neurologischer Erkrankungen. Stuttgart: Kohlhammer; 2012

[15] Lancaster E, Martinez-Hernandez E, Dalmau J. Encephalitis and antibodies to synaptic and neuronal cell surface proteins. Neurology 2011; 77: 179-189

[16] Baumgartner A, Rauer S, Mader I et al. Cerebral FDG-PET and MRI findings in autoimmune limbic encephalitis: correlation with autoantibody types. J Neurol 2013; 260: 27442753

[17] Endres D, Rauer S, Kern W et al. Psychiatric Presentation of Anti-NMDA Receptor Encephalitis. Front Neurol 2019; 10: 1086. doi: 10.3389/fneur.2019.01086 eCollection 2019

Bibliografie

PSYCH up2date 2020; 14: 460-467

DOI 10.1055/a-1045-8646

ISSN 2194-8895

(C) 2020. Thieme. All rights reserved.

Georg Thieme Verlag KG, Rüdigerstraße 14

70469 Stuttgart, Germany 